\title{
Heart rhythm complexity as predictors for the prognosis of end-stage renal disease patients undergoing hemodialysis
}

\author{
Hongyun Liư', Ping Zhan ${ }^{1}$, Jinlong Shi ${ }^{2}$ Minlu Hu', Guojing Wang ${ }^{1}$ and Weidong Wang ${ }^{\text {** }}$
}

\begin{abstract}
Background: Heart rhythm complexity, a measure of heart rate dynamics and a risk predictor in various clinical diseases, has not been systematically studied in patients with end-stage renal disease. The aim of this study is to investigate the heart rhythm complexity and its prognostic value for mortality in end-stage renal disease patients undergoing hemodialysis.
\end{abstract}

Methods: To assess heart rhythm complexity and conventional heart rate variability measures, 4-h continuous electrocardiography for a retrospective cohort of 202 ostensibly healthy control subjects and 51 hemodialysis patients with end-stage renal disease were analyzed. Heart rhythm complexity was quantified by the complexity index from the measurement of the multiscale entropy profile.

Results: During a follow-up of 13 months, 8 people died in the patient group. Values of either traditional heart rate variability measurements or complexity indices were found significantly lower in patients than those in healthy controls. In addition, the complexity indices (Area 1-5, Area 6-15 and Area 6-20) in the mortality group were significantly lower than those in the survival group, while there were no significant differences in traditional heart rate variability parameters between the two groups. In receiver operating characteristic curve analysis, Area 6-20 (AUC $=0.895, p<0.001$ ) showed the strongest predictive power between mortality and survival groups.

Conclusion: The results suggest that heart rhythm complexity is impaired for patients with end-stage renal disease. Furthermore, the complexity index of heart rate variability quantified by multiscale entropy may be a powerful independent predictor of mortality in end-stage renal disease patients undergoing hemodialysis.

Keywords: Complexity, Multiscale entropy, End-stage renal disease, Autonomic nervous system, Heart rate variability

\section{Background}

End-stage renal disease (ESRD), characterized by permanent loss of renal function, is the final stage in chronic kidney disease that becomes a global health problem with increasing prevalence [1]. Despite recent progress in medical management and dialysis therapy

\footnotetext{
*Correspondence: wang_weidong301@163.com

${ }^{1}$ Research Center for Biomedical Engineering, Medical Innovation \& Research Division, Chinese PLA General Hospital, Fuxing Road, Beijing 100853, China Full list of author information is available at the end of the article
}

technique, mortality rates remain high in ESRD patients undergoing hemodialysis. The major causes of ESRD are largely the same causes that contribute to cardiovascular disease [2]. In addition, sudden cardiac death is common in patients receiving hemodialysis and may account for about $30 \%$ of overall mortality, which could partly owe to the impairment of the autonomic nervous system (ANS) [3-5]. Identification of high-risk patients with ESRD is of clinical importance and considerable practical value in strengthening their treatment strategies. 
Patients with ESRD undergoing chronic hemodialysis is usually present a dysfunction of the ANS, exhibited as low parasympathetic/vagal modulation associated with high sympathetic modulation of the heart rate assessed by heart rate variability (HRV) analysis [6-8]. Several previous studies have used HRV parameters based on traditional linear HRV analysis to predict patient outcomes and found that lower HRV is related to an increased risk of cardiovascular and all-cause mortality in patients with ESRD receiving chronic hemodialysis [913]. However, the regulation of heart rate by the ANS is considered to be a non-linear physiological process with complexity. Recently, multiscale entropy (MSE) measures quantify multiple spatial and temporal scale complexity of heart rate dynamics, have been introduced as new risk stratifiers [14, 15]. At present, MSE has been extensively applied in the assessment of heart rhythm complexity in heart failure, stroke, myocardial infarction, epilepsy and other diseases for predicting patient outcomes [16-19]. Previous studies have also demonstrated that MSE parameters have greater predictive power than conventional HRV measures for risk stratification and prognosis $[19,20]$. Heart rhythm complexity impairment has been associated with adverse outcomes in clinical settings. However, its potential relation to the increased risk of death in ESRD patients undergoing hemodialysis may be underappreciated and need to be further elucidated. Therefore, the aim of the present study was to investigate the heart rate complexity derived from MSE analysis of long-term electrocardiograms (ECG) and its prognostic value for all-cause mortality in patients with ESRD receiving hemodialysis therapy.

\section{Methods}

\section{The study protocol and population}

Healthy database (THEW identification: E-HOL-030202-003) with 202 24-h ECG recordings and ESRD database (THEW identification: E-HOL-03-0202-016) contains 51 48-h long-term ECG recordings were selected from the Telemetric and Holter ECG Warehouse (THEW, http://thew-project.org/datab ases.htm) at University of Rochester. Raw ECG data, as well as automated beat annotations reviewed and adjudicated manually, are available in both databases. All human data were obtained retrospectively from completed, Institutional-Review-Board-approved clinical research studies with subject de-identification. These trials complied with the Declaration of Helsinki and all subjects signed informed consent documents.

The ESRD database comprises Holter recordings with a sampling frequency of $1000 \mathrm{~Hz}$ from 51 ESRD patients with a high risk of death. All 51 ESRD patients underwent high-resolution 12-lead 48-h continuous ECG monitoring, and THEW provides 12-lead raw ECG and heartbeat interval data. ESRD subjects receiving hemodialysis confirmed the history of diabetes or hypertension requiring treatment entered into this study. The ESRD patients were enrolled from February 13, 2009 to June 18, 2010, and they completed their 13-month follow-up evaluation. All patients Exclusion criteria included a history of chronic atrial fibrillation, with class I antiarrhythmic, pacemaker, implantable cardioverterdefibrillator device, cardiac resynchronization therapy device, female subject of childbearing potential not using medically prescribed contraceptive measures and subject unable to cooperate with the protocol due to dementia, psychological, or other related reason. The Healthy database comprises 24-h Holter recordings from 202 ostensibly healthy subjects. Subjects had (1) no overt cardiovascular disease or history of cardiovascular disorders; (2) no reported medications, history of high blood pressure and chronic illness, (3) a normal physical examination, (4) a 12-lead ECG showing sinus rhythm with normal waveforms (or a normal echocardiogram and normal ECG exercise testing in the presence of any questionable findings ECG changes). The ECG signals were recorded at a sampling frequency of $200 \mathrm{~Hz}$. In order to reduce the influence of gender and age on ECG parameters, we try to match each ESRD patient with a healthy control with the same gender and close age. After excluding the poor signal quality and incomplete ECG recordings, 51 ostensibly age-matched healthy control subjects were selected from 202 healthy subjects and eventually enrolled in the present study.

\section{ECG preprocessing}

All long-term ECG recordings were analyzed with Kubios (Kubios 2.2, University of Eastern Finland, Kuopio), on which $\mathrm{R}$ waves were detected and labeled automatically. Heart-beat interval between 300 and $2000 \mathrm{~ms}$, consecutive heart-beat interval differences $\leq 200 \mathrm{~ms}$, and prolongations or shortenings $\leq 20 \%$ than the average of five preceding sinus rhythm heart-beat intervals were considered as sinus rhythm QRS complexes [21]. Thereafter, automatic annotated results were carefully visual inspected and manually corrected by editing ectopic beats, arrhythmias and noise to suppress computational errors. Four-hour episodes of heart-beat intervals without naps and exercise within daytime (between 8 a.m. and 5 p.m.) were extracted from each recording for MSE and traditional HRV analysis $[18,19]$. The 4-h ECG recordings of ESRD patients used in the present study were selected after the ideal body weight to reduce the influence of volume overload on HRV. All ECG segments were selected from the same period to reduce the confounding effects that may occur due to sleep or diurnal rhythm. Furthermore, four-hour ECG segments of 
patients with ESRD after hemodialysis sessions while performing their usual daily activities were used for further analysis.

\section{Traditional HRV analysis}

Traditional techniques of HRV analysis are grouped into the time domain, frequency domain, and non-linear methods. The time-domain measures including mean heartbeat intervals (Mean RR), standard deviation of the heartbeat intervals (SDNN), square root of the mean of sum of squares of the differences between adjacent heartbeat intervals (RMSSD), and percentage of the absolute change in consecutive heartbeat interval exceeds $50 \mathrm{~ms}$ (pNN50) were calculated to represent the total variance and vagal modulation of heart rate [22]. Based on the Fast Fourier transform spectrum, the frequency domain measures were computed from the power spectral density estimate for each frequency band including absolute power values of very low frequency (VLF, $0.0033-0.04 \mathrm{~Hz}$ ), low frequency (LF, $0.04-0.15 \mathrm{~Hz}$ ), high frequency (HF, $0.15-0.40 \mathrm{~Hz}$ ) bands, total power (TP, $0.0033-0.40 \mathrm{~Hz}$ ) and LF/HF power ratio [22]. The VLF, LF, HF and TP were also transformed in natural logarithmic (ln) value. Five traditional non-linear measures were also taken into consideration to characterize the properties of HRV. SD1 denotes the short-term variability caused by respiration, whereas SD2 denotes the longterm variability with both calculated through the Poincaré plot method [23]. Approximate entropy (ApEn) quantifies the single-scale complexity or regularity of the HRV time series by measuring the unpredictability of fluctuation patterns, and more uncorrelated random HRV signals usually produce higher ApEn value [24]. As a technique for characterizing the nature of long-range correlations in time series, detrended fluctuation analysis (DFA) was applied in the present study to quantify slope $\alpha 1$ and $\alpha 2$ for characterizing the short-term and longterm fluctuations of HRV signal, respectively [25].

\section{MSE analysis}

The MSE technique was proposed to characterize complex structure of non-linear and non-stationary physiological signals in different temporal scales that ignored by traditional entropy methods. It comprises of two steps: 1) coarse-graining the time series in finite length into different time scales; 2) quantifying the degree of irregularity in each coarse-grained time series by sample entropy calculations $[14,15]$. The quantified entropy values of coarse-grained time series then are represented as the function of time scale factors to evaluate the complex structure of physiological time series, and the features of the MSE curve can be extracted for clinical categorization in several diseases [16-19]. In-depth, details of this methodology have been previously described
$[14,15]$. In the present study, the complexity index (CI) of the HRV time series were quantified by curve fitting and calculating the area between the MSE curve and the axis of scale factors $[18,19]$. The linear-fitted slope (Slope 5) and the area under the MSE curve between scales 1 and 5 (Area 1-5) were calculated to quantify the short-term complexity and to characterize the shortscale modulation pattern. Long time scale complexity was quantified by the fitted area under the MSE curve between scales 6 and 15 (Area 6-15) and between scales 6 and 20 (Area 6-20), respectively. Since low frequency drifts, high frequency non-stationarities and general hidded trends longer than $2 \mathrm{~h}$ may lead to incorrectly increased irregularity and diminished sequence matching manifesting unpredictable effects on calculated sample entropy values. Empirical mode decomposition (EMD) is suitable for decomposition of non-stationary, non-linear physiologic time series and possesses advantages over wavelet and Fourier analysis because it employs a fully adaptive approach derived by means of a sifting process. In order to remove such effects, we used empirical mode decomposition (EMD) method for raw HRV time series filtering before performing MSE [18, 19].

\section{Statistical analysis}

Clinical data and parameters of ECG recordings were presented as median (25th and 75th percentiles). Gaussian distribution and homogeneity of variance tests were applied to determine the distribution and homoscedasticity of sample data. As a result of the non-normal distribution and heterogeneity of variance of some sample data, continuous variables were compared between different groups by the Mann-Whitney $U$ test. For single predictive variable analysis using qualitative or categorical variables, Fisher's exact tests were applied for comparison between different groups. Correlations between clinical variables and independent factors that predicting all-cause death for ESRD patients were performed using Spearman's correlation tests. The receiver operating characteristics (ROC) curve was created based on the sensitivity and specificity of HRV measures in predicting all-cause death in ESRD patients undergoing hemodialysis. The area under the ROC curve (AUC) gave an estimate of the overall discriminate ability (AUC $=0.5$ indicates no discrimination and an $\mathrm{AUC}=$ 1.0 indicates a perfect diagnostic test). Statistical analyses were performed using SPSS version 20 software package (SPSS, Chicago, Ill, USA). The maximal hazards ratio and independent correlation of variables with mortality was determined by Cox regression analysis. Then, Kaplan-Meier event probability curves and log rank analysis of the dichotomized groups were obtained. For all statistical analysis, $p$ values were corrected by the false 
discovery rate (FDR) method for multiple comparisons and $p<0.05$ was considered significant.

\section{Results}

\section{Study population}

Eventually, a total of 51 ESRD patients and 51 ostensibly healthy control subjects were enrolled in the present study. After a follow-up period of around 13 months, 8 (15.7\%) patients died in the ESRD group. There were 4 cardiac-related deaths and the other 4 patients died for unknown reasons. As shown in Table 1, ideal body weight, body mass index (BMI) and pre-dialysis systolic blood pressure (BP_SYS) values were significantly higher in ESRD patients while the prevalence of smoking $(11.8 \%$ vs. $39.2 \%, p<$ 0.001) was lower in ESRD group. However, there were no significant differences in gender, age, height and predialysis diastolic blood pressure (BP_DIA) between the ESRD and healthy groups. Detail information on demographic data, clinical characteristics, and laboratory data prior to hemodialysis for ESRD patients including both survival and mortality groups are presented in Table 2. No clinical variable was significantly different between these two groups (survival and mortality).

Table 1 Demographic data and basic characteristics of ESRD patients and healthy control subjects

\begin{tabular}{|c|c|c|c|}
\hline Variables & $\begin{array}{l}\text { ESRD patients } \\
(n=51)\end{array}$ & $\begin{array}{l}\text { Healthy control subjects } \\
(n=51)\end{array}$ & $P$ value \\
\hline Male/female & $30 / 21$ & $24 / 27$ & 0.321 \\
\hline Age (year) & $57(52-67)$ & $58(52-65)$ & 0.859 \\
\hline Height (cm) & $169(162-177)$ & $168(159-175)$ & 0.451 \\
\hline Weight (kg) & $82(72-97)$ & $70(64-83)$ & $<0.001$ \\
\hline BMI $\left(\mathrm{kg} / \mathrm{m}^{2}\right)$ & $29.37(24.39-33.47)$ & $25.30(23.62-27.48)$ & $<0.001$ \\
\hline BP_SYS (mmHg) & $143(127-169)$ & $120(118-140)$ & $<0.001$ \\
\hline BP_DIA (mmHg) & $73(65-88)$ & $80(75-80)$ & 0.227 \\
\hline Smoking, n (\%) & $6(11.8)$ & $20(39.2)$ & 0.003 \\
\hline Diabetes Mellitus, n(\%) & $32(62.7 \%)$ & N.A & N.A \\
\hline Hypertension, n(\%) & $51(100 \%)$ & N.A & N.A \\
\hline LVEF (\%) & $60(55-65)$ & N.A & N.A \\
\hline Duration of dialysis (year) & $2(3-6)$ & N.A & N.A \\
\hline $\mathrm{Kt} / \mathrm{V}$ & $1.42(1.24-1.61)$ & N.A & N.A \\
\hline URR (\%) & $74(70-79)$ & N.A & N.A \\
\hline $\mathrm{nPCR}$ & $0.85(0.72-0.97)$ & N.A & N.A \\
\hline Sodium (mEq/dL) & $139(137-141)$ & N.A & N.A \\
\hline Potassium (mEq/dL) & $5.1(4.4-5.4)$ & N.A & N.A \\
\hline Chloride (mEq/dL) & $100(97-103)$ & N.A & N.A \\
\hline Bicarbonite (mEq/dL) & $26(22-29)$ & N.A & N.A \\
\hline Blood Urea Nitrogen (mg/dL) & $58(45-72)$ & N.A & N.A \\
\hline Creatinine (mg/dL) & $8.3(6.6-10.4)$ & N.A & N.A \\
\hline Glucose (mg/dL) & $106(88-137)$ & N.A & N.A \\
\hline Calcium (mg/dL) & $8.7(8.3-9.2)$ & N.A & N.A \\
\hline Phosphate (mg/dL) & $5.3(4.3-6.2)$ & N.A & N.A \\
\hline Calcium Phosphate Product & $44.7(35.6-51.1)$ & N.A & N.A \\
\hline Albumin (g/dL) & $4.0(3.8-4.2)$ & N.A & N.A \\
\hline Hemoglobin (g/dL) & $11.7(11.0-12.3)$ & N.A & N.A \\
\hline Hematocrit (\%) & $36.0(34.0-38.0)$ & N.A & N.A \\
\hline$\beta$-blocker, n (\%) & $34(66.7)$ & N.A & N.A \\
\hline CCB, n (\%) & $25(49.0)$ & N.A & N.A \\
\hline ACEI, n (\%) & $15(29.4)$ & N.A & N.A \\
\hline
\end{tabular}

ESRD end-stage renal disease, $B M I$ body mass index, $B P \_S Y S$ systolic blood pressure, $B P \_D I A$ diastolic blood pressure, N.A not applicable, $L V E F$ left ventricular ejection fraction, URR urea reduction ratio, $n P C R$ normalized protein catabolic rate, $C C B$ calcium channel blocker, $A C E I$ angiotensin-converting-enzyme inhibitor 
Table 2 Clinical data of the ESRD patients in survival and mortality groups

\begin{tabular}{|c|c|c|c|}
\hline Variables & $\begin{array}{l}\text { Survival } \\
(n=43)\end{array}$ & $\begin{array}{l}\text { Mortality } \\
(n=8)\end{array}$ & $P$ value \\
\hline Male/female & $25 / 18$ & $5 / 3$ & 1.000 \\
\hline Age (year) & $55(52-65)$ & $66(62-70)$ & 0.087 \\
\hline Height (cm) & $170(162-178)$ & $168(166-171)$ & 0.595 \\
\hline Weight (kg) & $83(72-101)$ & $76(65-93)$ & 0.223 \\
\hline $\mathrm{BMI}\left(\mathrm{kg} / \mathrm{m}^{2}\right)$ & 30.22 (24.39-34.29) & $26.28(24.09-31.76)$ & 0.214 \\
\hline BP_SYS (mmHg) & $141(127-169)$ & $146(123-183)$ & 0.866 \\
\hline BP_DIA (mmHg) & $77(69-83)$ & $71(58-88)$ & 0.517 \\
\hline Smoking, n (\%) & $5(11.6)$ & $1(12.5)$ & 1.000 \\
\hline Diabetes Mellitus, n (\%) & $25(58.1)$ & $7(87.5)$ & 0.672 \\
\hline Hypertension, n (\%) & $43(100)$ & $8(100)$ & 1.000 \\
\hline Duration of dialysis (year) & $3(2-6)$ & $3(2-7)$ & 0.793 \\
\hline LVEF (\%) & $60(55-64)$ & $62(51-85)$ & 0.370 \\
\hline $\mathrm{Kt} / \mathrm{N}$ & $1.42(1.24-1.61)$ & $1.44(1.25-1.64)$ & 0.776 \\
\hline URR (\%) & $74(70-79)$ & $75(69-80)$ & 0.640 \\
\hline $\mathrm{nPCR}$ & $0.85(0.69-0.96)$ & $0.95(0.76-1.05)$ & 0.158 \\
\hline Sodium (mEq/dL) & $139(137-141)$ & $139(137-142)$ & 0.886 \\
\hline Potassium (mEq/dL) & $5.0(4.4-5.3)$ & $5.2(4.5-5.5)$ & 0.357 \\
\hline Chloride (mEq/dL) & $100(97-103)$ & $100(97-103)$ & 0.897 \\
\hline Bicarbonite (mEq/dL) & $27(23-29)$ & $22(21-25)$ & 0.065 \\
\hline Blood Urea Nitrogen (mg/dL) & $56(43-70)$ & $70(50-89)$ & 0.214 \\
\hline Creatinine $(\mathrm{mg} / \mathrm{dL})$ & $8.3(6.6-9.5)$ & $9.0(6.6-11.0)$ & 0.595 \\
\hline Glucose (mg/dL) & $106(88-143)$ & $110(81-136)$ & 0.698 \\
\hline Calcium (mg/dL) & $8.8(8.4-9.2)$ & $8.6(6.8-9.1)$ & 0.232 \\
\hline Phosphate (mg/dL) & $5.2(3.9-6.0)$ & $5.6(4.8-6.6)$ & 0.249 \\
\hline Calcium Phosphate Product & $44.5(35.5-51.3)$ & $46.0(42.0-49.7)$ & 0.679 \\
\hline Albumin $(\mathrm{g} / \mathrm{dL})$ & $4.0(3.8-4.2)$ & $4.0(3.5-4.3)$ & 0.630 \\
\hline Hemoglobin (g/dL) & $11.7(10.9-12.3)$ & $11.6(11.2-12.7)$ & 0.688 \\
\hline Hematocrit (\%) & $36.0(33.0-38.0)$ & $37.0(36.0-39.0)$ & 0.076 \\
\hline$\beta$-blocker, n (\%) & $29(67.4)$ & $5(62.5)$ & 0.541 \\
\hline$C C B, n(\%)$ & $20(46.5)$ & $5(62.5)$ & 0.329 \\
\hline ACEI, n (\%) & $13(30.2)$ & $2(25.0)$ & 0.565 \\
\hline
\end{tabular}

ESRD end-stage renal disease, $L V E F$ left ventricular ejection fraction, URR urea reduction ratio, $n P C R$ normalized protein catabolic rate, $C C B$ calcium channel blocker, $A C E l$ angiotensin-converting-enzyme inhibitor

\section{Holter data}

The results of traditional HRV and MSE analyses in ESRD and healthy groups are presented in Table 3 and Fig. 1. For traditional HRV parameters and CI derived from the MSE profiles, ESRD patients had significantly lower SDNN, RMSSD, pNN50, VLF, LF, HF, TP, LF/HF, SD1, SD2, $\alpha 1$, Slope 5, Area 1-5, Area 6-15 and Area 6-20 in comparison to the healthy control subjects. In contrast, patients with ESRD had significantly higher ApEn and $\alpha 2$ values than those of healthy controls (all $p<0.05$, Table 3$)$. The ESRD patients exhibited significantly reduced entropy values over most of the time scales, except scale 1, 2, 13 and 14 (Fig. 1). In addition, all of the average MSE curves of ESRD group had a pattern of an initial decrease which is different from that of the healthy control group.

For all the analyzed traditional HRV analyses, there were no significant differences between the survival and mortality groups (all $p>0.05$, Table 4). For MSE analysis, the entropy values were significantly lower over different time scales in the mortality group (Fig. 1). Meanwhile, the mortality group had significantly lower CI including Area 1-5 (2.49 (1.79-3.68) vs. 4.11 (3.36$4.72), p=0.002)$, Area $6-15(7.39(6.27-9.92)$ vs. 11.92 
Table 3 Traditional HRV measurements and Cl over 4-h continuous ECG recordings for ESRD patients and healthy control subjects

\begin{tabular}{|c|c|c|c|}
\hline Variables & $\begin{array}{l}\text { ESRD patients } \\
(n=51)\end{array}$ & $\begin{array}{l}\text { Healthy control subjects } \\
(n=51)\end{array}$ & $P$ value \\
\hline \multicolumn{4}{|c|}{ Traditional HRV analysis } \\
\hline Mean RR (msec) & $779(733-858)$ & 754 (690-836) & 0.137 \\
\hline SDNN (msec) & $36(28-50)$ & $71(59-95)$ & $<0.001$ \\
\hline RMSSD (msec) & $12(8-15)$ & $18(13-29)$ & $<0.001$ \\
\hline pNN50 (\%) & $0.14(0.02-1.06)$ & $1.77(0.42-6.64)$ & $<0.001$ \\
\hline $\operatorname{VLF}\left(\mathrm{msec}^{2}\right)$ & $18(10-41)$ & $68(45-142)$ & $<0.001$ \\
\hline LF $\left(\mathrm{msec}^{2}\right)$ & $50(23-178)$ & $333(234-790)$ & $<0.001$ \\
\hline $\mathrm{HF}\left(\mathrm{msec}^{2}\right)$ & $34(14-65)$ & $106(46-270)$ & $<0.001$ \\
\hline TP $\left(\mathrm{msec}^{2}\right)$ & $109(55-280)$ & $511(376-1232)$ & $<0.001$ \\
\hline $\operatorname{lnVLF}\left(\mathrm{msec}^{2}\right)$ & $2.88(2.32-3.69)$ & $4.23(3.85-4.84)$ & $<0.001$ \\
\hline $\operatorname{lnLF}\left(\mathrm{msec}^{2}\right)$ & $3.91(3.17-5.17)$ & $5.81(5.46-6.61)$ & $<0.001$ \\
\hline InHF $\left(\mathrm{msec}^{2}\right)$ & $3.51(2.68-4.13)$ & $4.67(3.86-5.48)$ & $<0.001$ \\
\hline $\operatorname{lnTP}\left(\mathrm{msec}^{2}\right)$ & $4.69(4.10-5.62)$ & $6.24(5.94-7.11)$ & $<0.001$ \\
\hline LF/HF & $1.68(0.84-3.96)$ & $3.42(2.37-6.85)$ & $<0.001$ \\
\hline SD1 (msec) & $8(5-10)$ & $12(9-20)$ & $<0.001$ \\
\hline SD2 (msec) & $14(10-23)$ & $31(26-47)$ & $<0.001$ \\
\hline ApEn & $1.47(1.36-1.52)$ & $1.36(1.27-1.46)$ & $<0.001$ \\
\hline a1 & $1.07(0.83-1.26)$ & $1.32(1.15-1.49)$ & $<0.001$ \\
\hline a2 & $0.62(0.49-0.71)$ & $0.52(0.46-0.61)$ & 0.005 \\
\hline \multicolumn{4}{|l|}{ MSE analysis } \\
\hline Slope 5 & $0.04(-0.04-0.08)$ & $0.10(0.07-0.15)$ & $<0.001$ \\
\hline Area 1-5 & $3.90(3.29-4.49)$ & $4.39(3.95-5.24)$ & 0.004 \\
\hline Area 6-15 & $11.62(9.13-12.85)$ & $12.63(11.42-13.65)$ & 0.013 \\
\hline Area 6-20 & $18.10(14.34-20.28)$ & $19.79(18.19-21.70)$ & 0.017 \\
\hline
\end{tabular}

ESRD end-stage renal disease, HRV heart rate variability, Mean RR mean heartbeat intervals, SDNN standard deviation of the heartbeat intervals, RMSSD square root of the mean of sum of squares of the differences between adjacent heartbeat intervals, $p N N 50$ percentage of the absolute change in consecutive heartbeat interval exceeds $50 \mathrm{~ms}, V L F$ very low frequency, LF low frequency, HF high frequency, TP total power, ApEn approximate entropy, MSE multiscale entropy
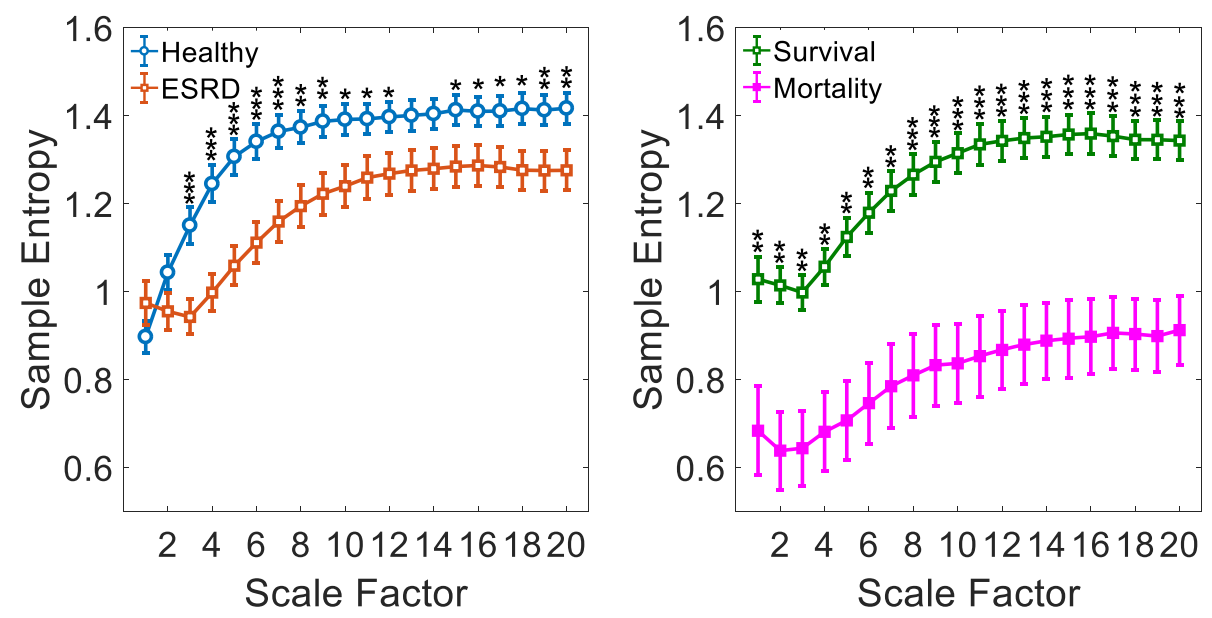

Fig. 1 The sample entropy over different time scales. The orange square open symbols represented the entropy of patients with ESRD, and the light blue open circles the entropy of healthy control subjects. The green open squares represented the entropy of the survival group after session hemodialysis treatment, and the pink solid squares the entropy of the mortality group after session hemodialysis treatment. Symbols represent the mean values of entropy for each group and bars represent the standard error ( $S E=S D / \sqrt{n}$, where $n$ is the number of subjects). ${ }^{*} p<0.05,{ }^{* *} p<0.01$, and ${ }^{* * *} p<0.001$ for comparison between groups 
Table 4 Traditional HRV measurements and Cl over 4-h continous ECG recordings for the study subjects in survival and mortality groups

\begin{tabular}{|c|c|c|c|}
\hline Variables & $\begin{array}{l}\text { Survival } \\
(n=43)\end{array}$ & $\begin{array}{l}\text { Mortality } \\
(n=8)\end{array}$ & $P$ value \\
\hline \multicolumn{4}{|c|}{ Traditional HRV analysis } \\
\hline Mean RR (msec) & 779 (732-885) & $795(753-852)$ & 0.846 \\
\hline SDNN (msec) & $34(26-48)$ & $43(35-53)$ & 0.143 \\
\hline RMSSD (msec) & $13(8-15)$ & $9(6-19)$ & 0.445 \\
\hline pNN50 (\%) & $0.14(0.02-1.06)$ & $0.07(0.01-3.97)$ & 0.917 \\
\hline $\operatorname{VLF}\left(\mathrm{msec}^{2}\right)$ & $21(10-51)$ & $11(4-22)$ & 0.117 \\
\hline $\mathrm{LF}\left(\mathrm{msec}^{2}\right)$ & $53(25-183)$ & $29(11-70)$ & 0.344 \\
\hline $\mathrm{HF}\left(\mathrm{msec}^{2}\right)$ & $34(16-65)$ & $16(7-102)$ & 0.526 \\
\hline TP $\left(\mathrm{msec}^{2}\right)$ & $141(70-311)$ & $67(32-155)$ & 0.271 \\
\hline $\operatorname{lnVLF}\left(\mathrm{msec}^{2}\right)$ & $3.02(2.36-3.82)$ & $2.43(1.76-2.79)$ & 0.119 \\
\hline $\operatorname{InLF}\left(\mathrm{msec}^{2}\right)$ & $3.96(3.23-5.20)$ & $3.37(2.77-4.07)$ & 0.351 \\
\hline $\operatorname{InHF}\left(\mathrm{msec}^{2}\right)$ & $3.52(2.85-4.10)$ & $2.76(2.31-4.26)$ & 0.533 \\
\hline InTP $\left(\mathrm{msec}^{2}\right)$ & $4.95(4.25-4.95)$ & $4.18(3.71-4.98)$ & 0.277 \\
\hline $\mathrm{LF} / \mathrm{HF}$ & $1.92(0.85-3.97)$ & $1.41(0.46-3.46)$ & 0.460 \\
\hline SD1 (msec) & $9(5-10)$ & $6(4-14)$ & 0.476 \\
\hline SD2 (msec) & $15(10-23)$ & $11(7-16)$ & 0.344 \\
\hline ApEn & $1.47(1.36-1.52)$ & $1.45(1.36-1.49)$ & 0.430 \\
\hline a1 & $1.07(0.83-1.27)$ & $1.04(0.57-1.21)$ & 0.476 \\
\hline$a 2$ & $0.64(0.52-0.71)$ & $0.59(0.34-0.73)$ & 0.445 \\
\hline \multicolumn{4}{|l|}{ MSE analysis } \\
\hline Slope 5 & $0.05(0.04-0.09)$ & $0.02(0.05-0.07)$ & 0.509 \\
\hline Area 1-5 & $4.11(3.36-4.72)$ & $2.49(1.79-3.68)$ & 0.002 \\
\hline Area 6-15 & $11.92(10.16-13.69)$ & 7.39 (6.27-9.92) & $<0.001$ \\
\hline Area 6-20 & $18.78(16.63-21.60)$ & 11.96 (9.84-15.54) & $<0.001$ \\
\hline
\end{tabular}

HRV heart rate variability, Mean RR mean heartbeat intervals, SDNN standard deviation of the heartbeat intervals, RMSSD square root of the mean of sum of squares of the differences between adjacent heartbeat intervals, $p N N 50$ percentage of the absolute change in consecutive heartbeat interval exceeds $50 \mathrm{~ms}, V L F$ very low frequency, $L F$ low frequency, $H F$ high frequency, $T P$ total power, ApEn approximate entropy, MSE multiscale entropy

(10.16-13.69), $p<0.001)$ and Area 6-20 (11.96 (9.84$15.54)$ vs. $18.78(16.63-21.60), \mathrm{p}<0.001)$ than those of survival group (Table 4). Furthermore, the above independent risk factors or predictors derived from MSE quantification did not significantly correlate with any clinical variables observed in the study (all $p>0.05$, Table 5).

The ROC curves of the predictive parameters (Area 1-5, Area 6-15 and Area 6-20) were depicted in Fig. 2. Area 6-20 (AUC $=0.895)$ showed the best overall discriminative power than Area 1-5 (AUC=0.858) and Area 6-15 (AUC=0.892) in the outcome prediction for ESRD patients receiving hemodialysis. Figure 3 showed Kaplan-Meier survival curves for all-cause death according to the contribution of Area 6-20. For the ESRD patients undergoing hemodialysis, survival seems to be
Table 5 Correlations between MSE parameters and clinical characteristics

\begin{tabular}{|c|c|c|c|}
\hline \multirow[t]{2}{*}{ Variables } & \multicolumn{3}{|c|}{ ESRD patients $(n=51)$} \\
\hline & Area 1-5 & Area 6-15 & Area $6-20$ \\
\hline Age (year) & 0.061 & -0.024 & -0.050 \\
\hline BP_SYS (mmHg) & -0.100 & -0.152 & -0.133 \\
\hline BP_DIA (mmHg) & -0.080 & 0.039 & 0.055 \\
\hline $\mathrm{BMI}\left(\mathrm{kg} / \mathrm{m}^{2}\right)$ & 0.017 & -0.152 & -0.134 \\
\hline LVEF (\%) & -0.058 & -0.061 & -0.069 \\
\hline Duration of dialysis (year) & 0.112 & 0.213 & 0.235 \\
\hline $\mathrm{Kt} / \mathrm{N}$ & 0.022 & -0.045 & -0.038 \\
\hline URR (\%) & 0.222 & 0.038 & 0.009 \\
\hline $\mathrm{nPCR}$ & -0.183 & -0.219 & -0.219 \\
\hline Sodium (mEq/dL) & 0.096 & 0.083 & 0.099 \\
\hline Potassium (mEq/dL) & -0.163 & -0.128 & -0.113 \\
\hline Chloride (mEq/dL) & -0.121 & -0.005 & 0.005 \\
\hline Bicarbonite (mEq/dL) & 0.197 & 0.106 & 0.111 \\
\hline Blood Urea Nitrogen (mg/dL) & -0.172 & -0.081 & -0.060 \\
\hline Creatinine (mg/dL) & 0.039 & 0.068 & 0.072 \\
\hline Glucose (mg/dL) & -0.130 & -0.129 & -0.117 \\
\hline Calcium (mg/dL) & 0.102 & 0.183 & 0.165 \\
\hline Phosphate (mg/dL) & -0.049 & -0.131 & -0.138 \\
\hline Calcium Phosphate Product & 0.019 & -0.098 & -0.112 \\
\hline Albumin (g/dL) & 0.118 & 0.087 & 0.071 \\
\hline Hemoglobin (g/dL) & -0.018 & -0.075 & -0.080 \\
\hline Hematocrit (\%) & -0.045 & -0.232 & -0.243 \\
\hline
\end{tabular}

related to whether the values of Area 6-20 were low (< 13.43). Cox regression analysis demonstrated that ESRD patients with Area $6-20<13.43$ had a higher risk of death $(4.8 \%$ vs. $66.7 \%)$ than those with Area 6-20 $\geq$ 13.43 (Hazard ratio $=13.61,95 \%$ CI: 2.74-67.77). However, no statistical risk factors were found in data of demographic characteristics, baseline clinical evaluation, or laboratory tests. The Area 6-20 may be a significant independent predictor of all-cause mortality for patients with ESRD, and when the cut-off value of Area 6-20 was set at 13.43 , the sensitivity and specificity were 88.4 and $75 \%$, respectively.

\section{Discussion}

To our knowledge, this is the first report to explore the predictive value of heart rhythm complexity quantified by MSE analysis in chronic hemodialysis ESRD patients. The results of the present study confirm that heart rhythm complexity and cardiac autonomic function are impaired in patients with ESRD. Importantly, the 


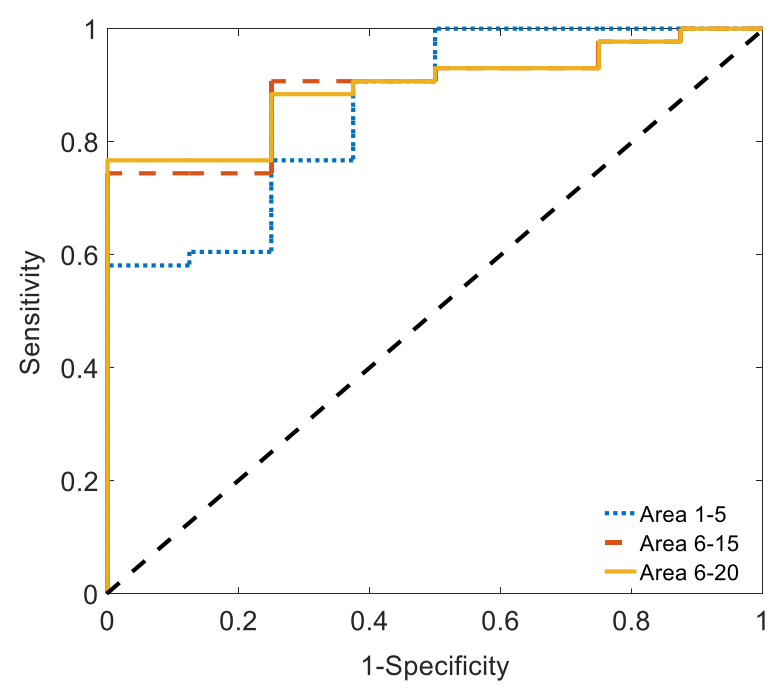

Fig. 2 Analysis of the discrimination power of the survival and mortality by receiver operating characteristic ( $R O C)$ curve analysis. The areas under the curve of Area 1-5, Area 6-15 and Area 6-20 were $0.858,0.892$ and 0.895 , respectively

deterioration of heart rhythm complexity seems to be related to a increased risk of subsequent motality. Specifically Area 6-20 among all the analyzed measures of HRV may be a predictor of increased mortality risk for allcause death in ESRD patients. Undergoing session hemodialysis, which is complementary to existing risk stratification strategies.

The dynamic balance of ANS plays a sophisticated and important role in maintaining the homeostasis of normal physiological processes to stay healthy in the human body [26]. Since HRV measures provide information on the degree of autonomic control of the heart rate, they are often used as a non-invasive and effective tool for assessment of the cardiac autonomic function and ANS state. Furthermore, low HRV is associated with mortality in various diseases [27-30]. In the present study, we found that all of the analyzed traditional HRV parameters, as well as conventional non-linear measures and CI, were significantly different between the ESRD and healthy control groups except for the Mean RR. Consistent with previous studies [10, 31-33], lowered HRV metrics and CI were observed in our study, which confirms that ESRD patients have impaired vagal control or cardiac autonomic function as well as heart rhythm complexity caused by disease pathology. Therefore, the multiscale method showed the phenomenon of heart rhythm decomplexification in ESRD patients, which contradicting the result obtained using the traditional ApEn algorithms.

DFA is often used to quantify the fractal correlation property of the HRV time series, and the short-term fractal scaling exponent $\alpha 1$ has been feasible to predict

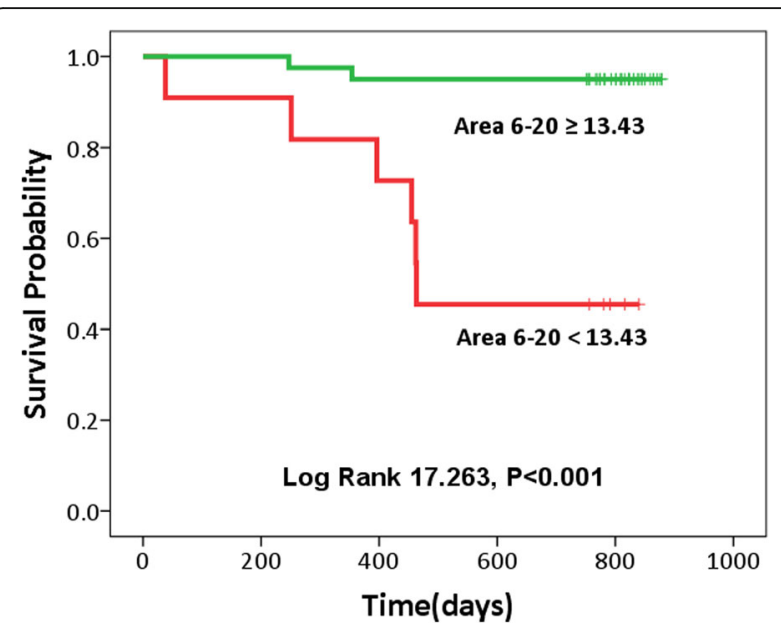

Fig. 3 Kaplan-Meier survival curves $(P<0.001)$ for all-cause mortality according to the the MSE parameters Area 6-20. The mortality for Area $6-20 \geq 13.43$ and Area $6-20<13.43$ were 4.80 and $66.7 \%$, respectively

mortality in various disease states [34-36]. Suzuki et al. showed that decreased $\alpha 1$ is an independent predictor for mortality in ESRD patients undergoing hemodialysis, and the addition of this metric to clinical risk factors significantly improves risk stratification [37]. In addition, Chiang et al. also found that increased short-term fractal scaling exponent $\alpha 1$ is an independent predictor for lower cardiac and total mortality in patients with ESRD receiving peritoneal dialysis [36]. However, Lin et al. demonstrated that there were no significant differences in fractal scaling exponent (both $\alpha 1$ and $\alpha 2$ ) for ESRD patients and those controls with normal renal function [38]. In the current study, we found that patients with ESRD had significantly lower $\alpha 1$ and higher $\alpha 2$ than those in healthy control subjects. This finding is consistent with previous reports of altered correlation properties under pathologic conditions [39]. Since antagonizing regulation of the sympathetic and vagal nerves on the heart is the physiological basis of the DFA method, the abnormal heart rate dynamics in ESRD patients evaluated by DFA are probably due to the co-activation of sympathetic and vagal outflow, which breakdown the intrinsic correlation property of HRV.

In this study, not only conventional non-linear measures like fractal scaling exponents and ApEn but all other traditional analyzed linear HRV metrics showed no independent predictive power for mortality in patients with ESRD receiving hemodialysis therapy. This finding is inconsistent with previous studies $[9,10,12$, $36,37]$. In addition, we observed no significant correlations of clinical risk factors with these HRV measures. The discrepancy between our findings and previous studies could be due to two main factors. The first, the ECG signals were recorded in free-running conditions, 
which may cause the measurements quantified by traditional linear and non-linear HRV analyses not to accurately assess the autonomic function. Additionally, different lengths of the HRV time series should also be taken into consideration for comparing the results of different studies. The second, the severity, cognitive performance, mental state and administration of drugs of ESRD patients can influence the results of HRV measures calculation, especially measurements (RMSSD, HF, $\alpha 1$, etc.) reflecting short-term variability and cardiac vagal regulation. The predictive ability of traditional linear and non-linear HRV parameters may be weakened and even absorbed by the associations between the above possible clinical risk factors and cardiac vagal impairment.

In contrast, heart rhythm complexity based on MSE appeared to enhance risk stratification in our study. CI Area 6-20 had the best performance for mortality prediction and is independent of clinical factors. This finding is consistent with previous studies regarding mortality using the MSE method. Norris et al. conducted a study to examine MSE parameters as predictors of trauma patient mortality in intensive care units. They concluded that MSE would be a robust predictor of trauma patient mortality in the face of variations in data density and duration [40]. The same group conducted afterward two larger studies, the results of which validated the above findings and confirmed that loss of heart rhythm complexity quantified by MSE could identify trauma patients at increased risk of subsequent hospital death $[41,42]$. Ho et al. investigated the prognostic value of parameters derived from MSE in patients with systolic heart failure. They found that MSE parameter Area 6-20 showed the strongest predictive power between survival and mortality groups among all the parameters [43]. In a study in patients receiving extracorporeal life support, Lin et al. also demonstrated that MSE metrics representing heart rhythm complexity were significantly associated with mortality [44]. This evidence showed the potential relationship between risk stratification, especially mortality and decomplexification of heart rate dynamics in patients with critical illnesses. Our results indicated that not only cardiac autonomic function but heart rhythm complexity were impaired in hemodialysis ESRD patients. Furthermore, the significant association between all-cause mortality and MSE index Area 6-20 implied a direct association between ESRD outcomes and heart rhythm complexity.

There are three limitations to our study. First, our findings are based on a retrospective, single-center, small sample size of ESRD patients with different medication administration. Therefore, it is difficult to discriminate between other possible contributing factors and to predict the possible effects of drugs on the cardiac autonomic function. The predictive ability of the MSE parameters might also be underpowered due to the small sample size. Second, we analyzed ECG recordings between hemodialysis sessions. The acute and chronic effects of hemodialysis on the heart rhythm complexity of patients with ESRD might be unpredictable. Third, the endpoint of our study was all-cause mortality, whether decomplexification of heart rate dynamics is associated with cardiac death is still needs to be elucidated. Our findings are preliminary, multicenter, sizeable and prospective studies are warranted to determine the relationship between mortality and heart rhythm complexity in ESRD patients undergoing hemodialysis treatment.

\section{Conclusions}

The present study suggests that impaired heart rhythm complexity is associated with poor prognosis in ESRD patients receiving hemodialysis treatment. Specifically, an Area $6-20<13.43$ seems to be a significant independent and powerful predictor of increased mortality risk for all-cause death. Heart rhythm complexity also provides an additional insight into risk stratification for ESRD patients. For ESRD patients with high risk of death, regular ECG monitoring combined with antiarrhythmic drugs and subcutaneous defibrillator may be valuable and useful for managing physician in developing all-cause mortality mitigation strategies.

\section{Abbreviationss \\ ESRD: End-stage renal disease; ANS: Autonomic nervous system; HRV: Heart rate variability; MSE: Multiscale entropy; ECG: Electrocardiograms; SDNN: Standard deviation of the heartbeat intervals; RMSSD: Root-mean- square of successive differences of normal-to-normal interbeat intervals; pNN50: The percentage of normal-to-normal intervals $>50 \mathrm{~ms}$ different from the previous interval; VLF: Very low frequency; LF: Low frequency; HF: High frequency; TP: Total power; ApEn: Approximate entropy; DFA: Detrended fluctuation analysis; EMD: Empirical mode decomposition; ROC: Receiver operating characteristic curve; AUC: Area under ROC curve; BMI: Body mass index}

\section{Acknowledgments}

The authors gratefully acknowledge the Telemetric and Holter ECG Warehouse of the University of Rochester (THEW) for their valuable work in sharing long-term ECG recordings of Healthy and ESRD database.

\section{Disclosures}

Not applicable.

\section{Authors' contributions}

HYL and JLS: responsible for the design and implementation of research. PZ, MLH and GJW: responsible for ECG data analysis and classification. HYL wrote the main body of the manuscript. WDW supervised the work. All authors read and approved the final manuscript.

\section{Funding}

This work was supported by the National Key Technology R\&D Program funded by Ministry of Science and Technology sponsored by the Chinese Government (No. 2016YFC1305703, No. 2015BAI01B08). The sponsors had no role in study design and conduct; data management, collection, and analysis; or preparation and submission of the manuscript. 


\section{Availability of data and materials}

The datasets used and/or analyzed during the current study are available from the corresponding author on reasonable request.

\section{Ethics approval and consent to participate}

All human data from Institutional-Review-Board-approved clinical research studies were archived at the University of Rochester Medical Center Telemetric and Holter ECG Warehouse (THEW) and made available for analyses by other investigators. A non-profit registration system to secure ethics of clinical trials and full written informed consent are provided by Telemetric and Holter ECG Warehouse of the University of Rochester with subject deidentification.

\section{Consent for publication}

Not applicable.

\section{Competing interests}

None of the authors have any conflict of interest to disclose. We confirm that we have read the Journal's position on issues involved in ethical publication and affirm that this report is consistent with those guidelines.

\section{Author details}

${ }^{1}$ Research Center for Biomedical Engineering, Medical Innovation \& Research Division, Chinese PLA General Hospital, Fuxing Road, Beijing 100853, China. ${ }^{2}$ Medical Big Data Center, Medical Innovation \& Research Division, Chinese PLA General Hospital, Fuxing Road, Beijing 100853, China.

Received: 3 January 2020 Accepted: 29 November 2020

\section{Published online: 09 December 2020}

\section{References}

1. Calderon-Margalit R, Golan E, Twig G, Leiba A, Tzur D, Afek A, Skorecki K, Vivante A. History of childhood kidney disease and risk of adult end-stage renal disease. N Engl J Med. 2018;378(5):428-38.

2. Wetmore JB, Collins AJ. Global challenges posed by the growth of endstage renal disease. Ren Replace Ther. 2016;2:15.

3. Chandra P, Sands RL, Gillespie BW, Levin NW, Kotanko P, Kiser M, Finkelstein F, Hinderliter A, Pop-Busui R, Rajagopalan S, Saran R. Predictors of heart rate variability and its prognostic significance in chronic kidney disease. Nephrol Dial Transplant. 2012;27(2):700-9.

4. Herzog CA, Mangrum JM, Passman R. Sudden cardiac death and dialysis patients. Semin Dial. 2010;21(4):300-7.

5. Hildreth CM. Prognostic indicators of cardiovascular risk in renal disease. Front Physiol. 2012;2:121

6. Converse RL, Jacobsen TN, Toto RD, Jost CMT, Cosentino F, Fouad-Tarazi F, Victor RG. Sympathetic overactivity in patients with chronic renal failure. N Engl J Med. 1992;327:1912-8.

7. Karayaylali I, San M, Kudaiberdieva G, Niyazova-Karben Z, Seyrek N, Balal M, Paydas S, Sagliker Y. Heart rate variability, left ventricular functions, and cardiac autonomic neuropathy in patients undergoing chronic hemodialysis. Ren Fail. 2003;25(5):845-53.

8. Tong YQ, Hou HM. Alteration of heart rate variability parameters in nondiabetic hemodialysis patients. Am J Nephrol. 2007;27(1):63-9.

9. Hayano J, Takahashi H, Toriyama T, Mukai S, Okada A, Sakata S, Yamada A, Ohte N, Kawahara $\mathrm{H}$. Prognostic value of heart rate variability during longterm follow-up in chronic haemodialysis patients with end-stage renal disease. Nephrol Dial Transpl. 1999;14(6):1480-8.

10. Fukuta H, Hayano J, Ishihara S, Sakata S, Mukai S, Ohte N, Ojika K, Yagi K, Matsumoto H, Sohmiya S, Kimura G. Prognostic value of heart rate variability in patients with end-stage renal disease on chronic haemodialysis. Nephrol Dial Transpl. 2003;18(2):318-25.

11. Pei J, Tang W, Li LX, Su CY, Wang T. Heart rate variability predicts mortality in peritoneal dialysis patients. Ren Fail. 2015;37(7):1132-7.

12. Oikawa K, Ishihara R, Maeda T, Yamaguchi K, Koike A, Kawaguchi H, Tabata $\mathrm{Y}$, Murotani $\mathrm{N}$, Itoh $\mathrm{H}$. Prognostic value of heart rate variability in patients with renal failure on hemodialysis. Int J Cardiol. 2009;131(3):370-7.

13. Kida N, Tsubakihara Y, Kida H, Ageta S, Arai M, Hamada Y, Matsuura N. Usefulness of measurement of heart rate variability by holter ecg in hemodialysis patients. BMC Nephrol. 2017;18(1):8.

14. Costa M, Goldberger AL, Peng CK. Multiscale entropy analysis of complex physiologic time series. Phys Rev Lett. 2002;89:068102.
15. Costa M, Goldberger AL, Peng CK. Multiscale entropy analysis of biological signals. Phys Rev E Stat Nonlinear Soft Matter Phys. 2005;71:021906.

16. Tang SC, Jen HI, Lin YH, Hung CS, Jou WJ, Huang PW, Shieh JS, Ho YL, Lai DM, Wu AY, Jeng JS, Chen MF. Complexity of heart rate variability predicts outcome in intensive care unit admitted patients with acute stroke. J Neurol Neurosurg Psychiatry. 2015;86:95-100.

17. Costa MD, Healey JA. Multiscale entropy analysis of complex heart rate dynamics: discrimination of age and heart failure effects. Computers In Cardiology IEEE. 2003;30:705-8.

18. Liu HY, Yang Z, Meng FG, Guan YG, Ma YS, Liang SL, Lin JL, Pan LS, Zhao MM, Qu W, Hao HW, Luan GM, Zhang JG, Li LM. Impairment of heart rhythm complexity in patients with drug-resistant epilepsy: an assessment with multiscale entropy analysis. Epilepsy Res. 2017;138:11-7.

19. Lin YH, Wu VC, Lo MT, Wu XM, Hung CS, Wu KD, Lin C, Ho YL, Stowasser M, Peng CK. Reversible heart rhythm complexity impairment in patients with primary aldosteronism. Sci Rep. 2015;5:11249.

20. Liu HY, Yang Z, Meng FG, Huang L, Qu W, Hao HW, Zhang JG, Li LM. Chronic vagus nerve stimulation reverses heart rhythm complexity in patients with drug-resistant epilepsy: an assessment with multiscale entropy analysis. Epilepsy Behav. 2018;83:168-74.

21. Clifford GD, MCSharry PE, Tarassenko L. Characterizing artifact in the normal human 24-hour RR time series to aid identification and artificial replication of circadian variations in human beat to beat heart rate using a simple threshold. Comput Cardiol. 2002;29:129-32.

22. Task F. Heart rate variability: standards of measurement, physiological interpretation, and clinical use. Circulation. 1996;93:1043-65.

23. Brennan M, Palaniswami M, Kamen P. Do existing measures of Poincare plot geometry reflect nonlinear features of heart rate variability. IEEE Trans Biomed Eng. 2001;48(11):1342-7.

24. Richman JA, Moorman JR. Physiological time-series analysis using approximate entropy and sample entropy. Am J Physiol Heart Circ Physiol. 2000;278:H2039-49.

25. Peng CK, Havlin S, Stanley HE, Goldberger AL. Quantification of scaling exponents and crossover phenomena in nonstationary heartbeat time series. Chaos. 1995;5:82-7.

26. Riganello F, Garbarino S, Sannita WG. Heart rate variability, homeostasis, and brain function: a tutorial and review of application. J Psychophysiol. 2012;26: 178-203.

27. Al-Zaiti SS, Pietrasik G, Carey MG, Alhamaydeh M, Canty JM, Fallavollita JA. The role of heart rate variability, heart rate turbulence, and deceleration capacity in predicting cause-specific mortality in chronic heart failure. J Electrocardiol. 2019;52:70-4.

28. de Castilho FM, Ribeiro ALP, Nobre V, Barros G, de Sousa MR. Heart rate variability as predictor of mortality in sepsis: a systematic review. PLoS One. 2018;13(9):e0203487.

29. Karimi MM, Setarehdan SK, Motie Nasrabadi A, Hajinasrollah E. Non-linear feature extraction from HRV signal for mortality prediction of ICU cardiovascular patient. J Med Eng Technol. 2016:40(3):87-98.

30. Bauer A, Kantelhardt JW, Barthel P, Schneider R, Mäkikallio T, UIm K, Hnatkova K, Schömig A, Huikuri H, Bunde A, Malik M, Schmidt G. Deceleration capacity of heart rate as a predictor of mortality after myocardial infarction: cohort study. Lancet. 2006;367:1674-81.

31. Rubinger D, Backenroth R, Sapoznikov D. Restoration of baroreflex function in patients with end-stage renal disease after renal transplantation. Nephrol. Dial. Transpl. 2009;24:1305-13.

32. Celik A, Melek M, Yuksel S, Onrat E, Avsar A. Cardiac autonomic dysfunction in hemodialysis patients: the value of heart rate turbulence. Hemodial Int. 2011;15:193-9.

33. Steinberg AA, Mars RL, Goldman DS, Percy RF. Effect of end-stage renal disease on decreased heart rate variability. Am J Cardiol. 1998;82:1156-8.

34. Ho KK, Moody GB, Peng CK, Mietus JE, Larson MG, Levy D, Goldberger AL. Predicting survival in heart failure case and control subjects by use of fully automated methods for deriving nonlinear and conventional indices of heart rate dynamics. Circulation. 1997;96:842-8.

35. Tapanainen JM, Thomsen PE, Kober L, Torp-Pedersen C, Mäkikallio TH, Still AM, Lindgren KS, Huikuri HV. Fractal analysis of heart rate variability and mortality after an acute myocardial infarction. Am J Cardiol. 2003;90:347-52.

36. Chiang JY, Huang JW, Lin LY, Chang CH, Chu FY, Lin YH, Wu CK, Lee JK, Hwang JJ, Lin JL, Chiang FT. Detrended fluctuation analysis of heart rate dynamics is an important prognostic factor in patients with end-stage renal disease receiving peritoneal dialysis. PLoS One. 2016;11(2):e0147282. 
37. Suzuki M, Hiroshi T, Aoyama T, Tanaka M, Ishii H, Kisohara M, lizuka N, Murohara T, Hayano J. Nonlinear measures of heart rate variability and mortality risk in hemodialysis patients. Clin J Am Soc Nephrol. 2012;7(9): 1454-60.

38. Lin YH, Lin C, Ho YH, Wu VC, Lo MT, Hung KY, Liu LY, Lin LY, Huang JW, Peng CK. Heart rhythm complexity impairment in patients undergoing peritoneal dialysis. Sci Rep. 2016;6:28202.

39. Peng CK, Havlin S, Hausdorff JM, Mietus JE, Stanley HE, Goldberger AL. Fractal mechanisms and heart rate dynamics. Long-range correlations and their breakdown with disease. J. Electrocardiol. 1995;28:59-65.

40. Norris PR, Anderson SM, Jenkins JM, Williams AE, Morris JA. Heart rate multiscale entropy at three hours predicts hospital mortality in 3,154 trauma patients. Shock. 2008;30(1):17-22

41. Norris PR, Stein PK, Morris JA. Reduced heart rate multiscale entropy predicts death in critical illness: a study of physiologic complexity in 285 trauma patients. J Crit Care. 2008;23(3):399-405.

42. Riordan WP, Norris PR, Jenkins JM, Morris JA. Early loss of heart rate complexity predicts mortality regardless of mechanism, anatomic location or severity of injury in 2178 trauma patients. J Surg Res. 2009;156(2):283-9.

43. Ho YL, Lin C, Lin YH, Lo MT. The prognostic value of non-linear analysis of heart rate variability in patients with congestive heart failure--a pilot study of multiscale entropy. PLoS One. 2011;6(4):e18699.

44. Lin YH, Huang HC, Chang YC, Lin C, Lo MT, Liu LY, Tsai PR, Chen YS, Ko WJ, Ho YL, Chen MF, Peng CK, Buchman TG. Multi-scale symbolic entropy analysis provides prognostic prediction in patients receiving extracorporeal life support. Crit Care. 2014;18(5):548.

\section{Publisher's Note}

Springer Nature remains neutral with regard to jurisdictional claims in published maps and institutional affiliations.

Ready to submit your research? Choose BMC and benefit from:

- fast, convenient online submission

- thorough peer review by experienced researchers in your field

- rapid publication on acceptance

- support for research data, including large and complex data types

- gold Open Access which fosters wider collaboration and increased citations

- maximum visibility for your research: over $100 \mathrm{M}$ website views per year

At $\mathrm{BMC}$, research is always in progress.

Learn more biomedcentral.com/submissions 